\title{
BMJ Open Kindy Moves: a protocol for establishing the feasibility of an activity-based intervention on goal attainment and motor capacity delivered within an interdisciplinary framework for preschool aged children with cerebral palsy
}

\author{
Dayna Pool (D , , ${ }^{1,2}$ Catherine Elliott, ${ }^{1,3}$ Healthy Strides Research Advisory Council
}

To cite: Pool D, Elliott C Healthy Strides Research Advisory Council. Kindy Moves: a protocol for establishing the feasibility of an activitybased intervention on goal attainment and motor capacity delivered within an interdisciplinary framework for preschool aged children with cerebral palsy. BMJ Open 2021;11:e046831. doi:10.1136/ bmjopen-2020-046831

- Prepublication history for this paper is available online. To view these files, please visit the journal online (http://dx.doi. org/10.1136/bmjopen-2020046831).

Received 10 November 2020 Accepted 03 August 2021

Check for updates

(C) Author(s) (or their employer(s)) 2021. Re-use permitted under CC BY-NC. No commercial re-use. See rights and permissions. Published by BMJ.

For numbered affiliations see end of article.

Correspondence to

Dr Dayna Pool;

Dayna.Pool@curtin.edu.au

\section{ABSTRACT}

Introduction Preschool aged children with cerebral palsy (CP) and like conditions are at risk of performing below their peers in key skill areas of school readiness. Kindy Moves was developed to support school readiness in preschool aged children with CP and like conditions that are dependent on physical assistance and equipment throughout the day. The primary aims are to determine the feasibility of motor-based interventions that are functional and goal directed, adequately dosed and embedded into a play environment with interdisciplinary support to optimise goal-driven outcomes.

Methods and analysis Forty children with CP and like conditions aged between 2 and 5 years with a Gross Motor Function Classification System (GMFCS) level of III-V or equivalent, that is, dependent on physical assistance and equipment will be recruited in Western Australia. Participants will undertake a 4-week programme, comprised three, 2-hour sessions a week consisting of floor time, gross motor movement and play (30 min), locomotor treadmill training (30 min), overground walking in gait trainers (30 min) and table-top activities (30 min). The programme is group based with 3-4 children of similar GMFCS levels in each group. However, each child will be supported by their own therapist providing an interdisciplinary and goal directed approach. Primary outcomes of this feasibility study will be goal attainment (Goal Attainment Scale) and secondary outcomes will include Canadian Occupational Performance Measure, 10 metre walk test, Children's Functional Independence Measure, Sleep Disturbance Scale, Infant and Toddler Quality of Life Questionnaire, Peabody Developmental Motor Scale and Gross Motor Function Measure. Outcomes will be assessed at baseline, post intervention (4 weeks) and retention at the 4-week follow-up.

Ethics and dissemination Ethical approval was obtained from Curtin University Human Ethics Committee (HRE2019 0073). Results will be disseminated through published manuscripts in peer-reviewed journals, conference presentations and public seminars for stakeholder groups.
Strengths and limitations of this study

- To our knowledge, this will be the first trial to evaluate the feasibility of a goal directed, activity-based and interdisciplinary programme to support schoolreadiness in preschool aged children with cerebral palsy (CP) and like conditions that rely on physical assistance and equipment.

- Kindy Moves is designed to develop motor-based capacity for children with $\mathrm{CP}$ and like conditions that rely on physical assistance and equipment by integrating locomotor treadmill training into a playbased environment. This has been identified in previous research where there are limited interventions available for children that rely on physical assistance and equipment.

- The trial protocol was designed in partnership with consumers and will be delivered through a community-based organisation.

- The multidisciplinary nature of the programme will make it difficult to differentiate between the effects of the individual elements of the programme.

Trial registration number Australian New Zealand Clinical Trials Registry (ACTRN12619000064101p).

\section{INTRODUCTION}

Early childhood is considered to be the most important developmental phase throughout the lifespan. ${ }^{1}$ It is widely documented that investments in early intervention yield greater economic rate of return when compared with investments later in childhood. ${ }^{2-4}$ Preschool attendance is strongly associated with developmental vulnerability at school entry. ${ }^{5}$ This highlights the significance of preschool programmes which have been shown to 
provide both short-term and long-term benefits on health, learning, development and well-being. ${ }^{5}$ The school readiness framework provides a structured understanding of the individual strength and vulnerability profiles of preschool aged children in the key skill areas of health and physical development, emotional well-being, social competence, approaches to learning, communication, cognitive skills and general knowledge ${ }^{67}$ Failure to intervene effectively in these key skill areas during the early years impacts across the lifespan. ${ }^{5}$ Therefore, identifying children who are at risk of performing below their peers in these key skill areas can ensure that the necessary supports and early intervention strategies can be implemented to optimise developmental outcomes and a successful transition into school.

Children at risk of performing below their peers at school include those with motor impairments that result from cerebral palsy (CP) or like conditions. ${ }^{8}{ }^{9} \mathrm{CP}$ is the most common cause of physical disability in childhood, ${ }^{10}{ }^{11}$ with nearly $40 \%$ of children dependent on physical assistance and equipment throughout the day ${ }^{10}$ and classified within the Gross Motor Function Classification System (GMFCS) as being levels III, IV and V. ${ }^{12}$ Like conditions are where there are also disturbances of movement and posture that can result from conditions that affect the central and peripheral nervous systems with causes ranging from genetic disorders, developmental or congenital abnormalities. ${ }^{13}{ }^{14}$ Children with CP like conditions can also experience motor limitations that similarly result in a dependence on physical assistance and equipment throughout the day. Given the higher prevalence of $\mathrm{CP}$ in childhood, recommendations in the current body of evidence commonly relates to CP only, but the growing trend towards a 'top-down' approach means that clinically, interventions employed for children with $\mathrm{CP}$ can also be used to inform strategies for like conditions. ${ }^{15}$ Collectively, mobility restrictions in this group of children is a barrier for school readiness and participation and as such, warrants the need for the development and implementation of interventions that focus on a 'top-down' approach for meaningful improvement in functional skills. ${ }^{716}$

The common thread of effective paediatric functional interventions for children with $\mathrm{CP}$ are interventions that are not only adequate dosed to achieve functional goals but also contain the essential active ingredients for motor skill acquisition. Interventions that are highly dosed and provided with intermittent or 'burst' schedules have shown greater likelihood of motor skill attainment when compared with continuous schedules with weekly sessions. ${ }^{17}$ The threshold of adequate dosage is yet to be defined with some models using dosages of 90 hours delivered over $2-3$ weeks, ${ }^{18}$ to models that include at least three sessions a week. ${ }^{17} 19$ The threshold for upper limb training for children with CP has suggested a dosage of between 15 and 25 hours for addressing three functional goals ${ }^{20}$ and for functional mobility training, a dosage of 18 hours delivered over 6 weeks has shown improvements in motor function. ${ }^{21}$ Beyond intervention dosage, research strongly supports the need for interventions to contain the essential active ingredients for improved motor ability. ${ }^{22}{ }^{23}$ This includes interventions that focus on the activity and participation level of the International Classification of Functioning - Child and Youth (ICF-CY), ${ }^{24}$ are task specific and goal directed, focused on function not normality, context specific and require active child involvement in order to achieve functional goals. ${ }^{22}$ At the centre of these models, practicality must be considered particularly with regards to costs in both time and resources which ultimately affects research translation into practice. Therapeutic interventions need to balance the importance of being adequately dosed to optimise outcomes with the impact of appointments on immediate and long-term family stress, fatigue and burden. ${ }^{17}$

A collaborative interdisciplinary approach has the advantage of intentionally blurring the traditionally concrete disciplinary boundaries. ${ }^{25}$ The adoption of this approach enables a range of expertise and skills that can be used within a single intervention. Such an approach is focused through a strengths-based lens and centred on meaningful goal-directed outcomes rather than discrete discipline specific outcomes only. ${ }^{25-29}$ As noted earlier, school readiness encompasses a range inter-related key skill areas, highlighting the importance of a context specific interdisciplinary approach. Early intervention strategies and international recommendations for children with CP strongly support the need for therapies to be delivered within the home context and this is vitally important for babies and toddlers. ${ }^{30}$ However, the preparation for school (including kindergarten or preschool) requires a context specific intervention. Therefore, an intervention that is delivered in a context that mirrors a school environment harnessing play within a group setting and set outside of the home is an important transition and consideration for school readiness. Play that is set within a group naturally involves multiple peer interactions, with improvements in some key skill areas of school readiness such as gains in expressive and receptive language,${ }^{31}$ turntaking, sharing and initiation of peer interaction ${ }^{32}$ having been observed. As such, a school readiness programme that includes play within a group context would be an important feature of the intervention.

Though it has been established that more mobile children have increased levels of participation, ${ }^{33-41}$ there is a paucity of effective motor-based interventions available for preschool aged children with CP and like conditions that are dependent on physical assistance and equipment throughout the day. ${ }^{42-44}$ Locomotor treadmill training, that is, LTT (includes partial body weight supported training and overground gait training) has shown promising improvements in both school-aged children with CP classified within GMFCS levels III, IV and V as well as in children as young as 4 years of age ${ }^{45-49}$ Beyond the diagnosis of children with CP, current evidence of LTT suggests accelerated motor development in preschool aged children with developmental delay. ${ }^{50}$ However, 
the dosage remains unclear with improvements in motor function being reported with as little as a 'burst' of training consisting of three, 1-hour sessions over 4 weeks. ${ }^{40}$ Given the potential for accelerated motor development with LTT, the range of key skill areas associated with school readiness that can be supported with an interdisciplinary team through the vehicle of play within a group, ${ }^{51}$ and the suggested dosages from previous studies on motor improvements, ${ }^{2049}$ it would be important to test the feasibility of an adequately dosed LTT in preschool aged children with CP and CP like conditions.

Therefore, within the context of supporting school readiness in children that are dependent on physical assistance and equipment throughout the day with $\mathrm{CP}$ and CP like conditions, motor-based interventions that are functional and goal directed, adequately dosed and embedded into a play environment with interdisciplinary support has the potential to optimise goal-driven outcomes. ${ }^{27} 28{ }^{52-55}$ This study aims to determine if such an intervention is feasible for preschool aged children with CP and CP like conditions that are dependent on physical assistance and equipment throughout the day, in improving functional goal attainment and motor capacity.

\section{METHODS}

\section{Aims and hypotheses}

The main aim of the proposed study is to determine the feasibility of the Kindy Moves programme (dosage of 24 hours) in improving goal attainment and motor capacity in children with CP and CP like conditions aged between 2 and 5 years. This feasibility trial will be tested in children with CP and CP like conditions that are classified within GMFCS levels III-V that rely on daily physical assistance and equipment.

The feasibility domains that will be assessed are based on the Bowen et al framework ${ }^{56}$ with acceptability and suitability (the extent to which Kindy Moves is judged to be suitable to parents and participants and their perceptions of its utility beyond the research), motivations for participating (the extent to which Kindy Moves is of interest to participants and their families) and practicality (the personal and environmental barriers and facilitators that affect the implementation and provision of Kindy Moves) assessed at post-treatment. A semi-structured interview with parents of the children attending the programme will be used to assess the feasibility domains with questions based on the F-words in childhood disability. ${ }^{57}$

Limited-efficacy testing is another feasibility domain and this will be assessed using objective measures to determine if Kindy Moves shows promise to be successful and effective in marginally ambulant and non-ambulant children with neurological disorders. ${ }^{56}$ For this domain, the primary hypothesis is that Kindy Moves will improve goal attainment on the Goal Attainment Scale (GAS) to a T-score of $50^{58}$ at T2 (after the 4-week programme) with retention at T3 (4 weeks after the conclusion of the programme) when compared with baseline (T1). The secondary hypotheses are that Kindy Moves will improve perceived performance and satisfaction in activity and participation goals by a mean difference of two points on the Canadian Occupational Performance Measure $(\mathrm{COPM}),{ }^{59}$ indoor walking speed on the 10 -metre walk test $(10 \mathrm{mWT})$ by $0.1 \mathrm{~m} / \mathrm{s},{ }^{60}$ functional independence on the Children's Functional Independence Measure (WeeFIM) ${ }^{61}$ fine motor skills on the Peabody Developmental Motor Scale Version 2 (PDMS-2), ${ }^{62}$ sleep behaviour and disturbances on the Sleep Disturbance Scale for Children ${ }^{63}$ and parent-reported quality of life on the Infant and Toddler Quality of Life ${ }^{64}$ at T2 (after the 4-week programme) with retention at T3 (4 weeks after the conclusion of the programme) when compared with baseline (T1). Given that $\mathrm{CP}$ is the most common cause of physical disability we also hypothesise that children will CP will improve their gross motor function on the Gross Motor Function Measure-GMFM-66 by 3 points. ${ }^{65}$

\section{Ethics}

Human ethics approval has been obtained from the Human Research Ethics Committees (HREC) at Curtin University, Perth Australia. Written and informed parent/guardian consent will be obtained prior to study commencement by the chief investigator. The study protocol is reported according to the Standard Protocol Items: Recommendations for Interventional Trials guidelines. Any changes in study protocol will be reported to the Australian New Zealand Clinical Trials Registry and HREC.

\section{Study sample and recruitment}

Recruitment will occur through The Healthy Strides Foundation's Facebook and Instagram pages. The Healthy Strides Foundation is a community-based not-for-profit organisation that provides intensive, multidisciplinary therapy for children with neurological conditions and injuries in Perth, Australia. After parents have read the eligibility criteria on the social media platforms, parents can complete an online form which will help determine eligibility. This initial self-referring online screening form will require parents to describe (selecting from prewritten options) how their child moves around the home and community and their child's hand function and communication development. Once reviewed, a phone screen will occur with the chief investigator to further clarify eligibility and provide an opportunity to discuss the study and their child's potential involvement. If the child meets the criteria, the participant information sheet will be sent electronically to parents and a baseline (T1) assessment scheduled. At the baseline assessment, confirmation of eligibility will be established with the consent form signed and witnessed. The study will run from March 2019 to December 2021. Due to the disruption to recruitment that occurred during COVID-19 restrictions in 2020, recruitment will continue throughout 2021. 


\section{INCLUSION AND EXCLUSION CRITERIA}

Participant inclusion criteria include children aged between 2 and 5 years, with CP or a CP like condition that results in functional mobility described as GMFCS levels III, IV and V or for non-CP conditions, are dependent on physical assistance and equipment throughout their day. Children must also have identified functional multidisciplinary goals in the area of mobility, communication or socialisation with peers and functional upper limb skills. Exclusion criteria include uncontrolled seizure disorder (defined as a seizure disorder that does not consistently respond to medical treatments and frequently (>two times per month) requires the administration of rescue medication and emergency call for the ambulance), orthopaedic surgery in the past 6 months, unstable hip subluxation or have engaged in LTT in the past month.

\section{Sample size determination}

Sample size for this single group feasibility trial is based on within group differences for the primary outcome measure GAS. A sample size of 34 participants was determined with a large effect size $(\mathrm{d}=1.0)$ hypothesised on the GAS t-score ( $80 \%$ power; two-sided test at $\mathrm{p}<0.05)$. To account for attrition, 40 children will be recruited.

Eligible children: Cerebral palsy or cerebral palsy like conditions, dependent on physical assistance and equipment. 2- 5 years of age, multidisciplinary goals. No orthopaedic surgery past 6 months or locomotor training last 4 weeks, uncontrolled seizure disorder or unstable hip subluxation

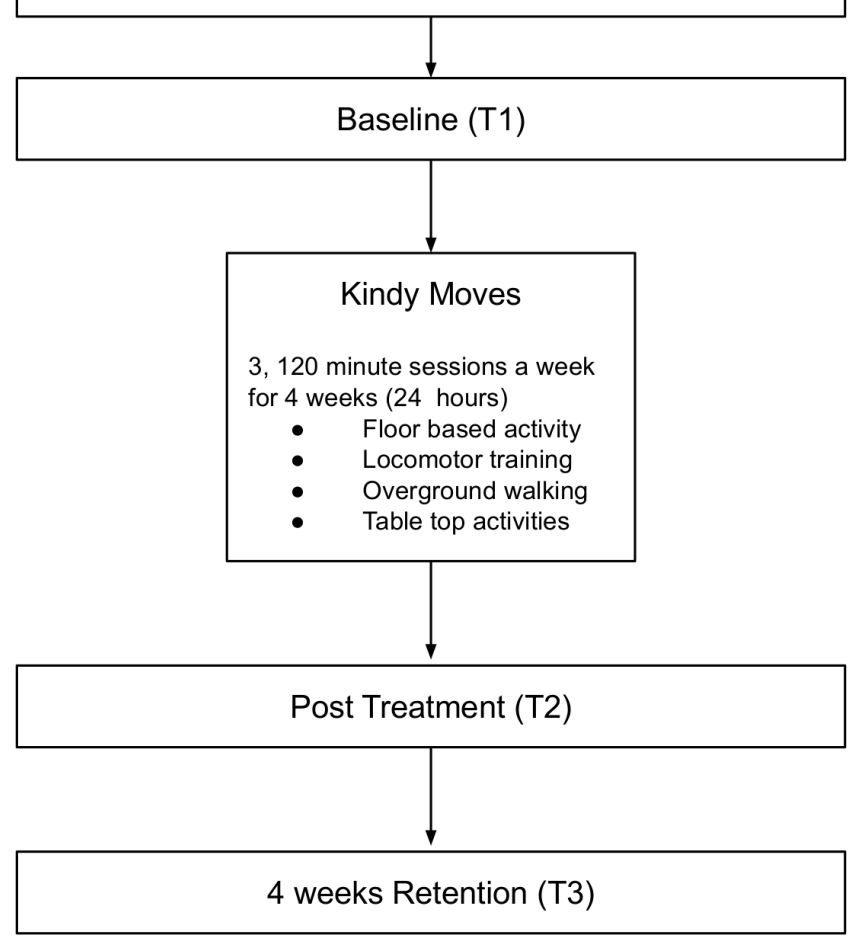

\section{Blinding}

The GMFM and PDMS-2 will be video recorded and scored by a blinded physiotherapist and occupational therapist respectively who will be unaware of the order of the videos being filmed (ie, T1, T2 or T3). The qualitative interviews will be conducted by an independent interviewer.

\section{Safety and adverse events}

To monitor any adverse events, parents will be questioned by the team at the beginning of each session. All events will be reported to the chief investigator and recorded on a database with any major events referred to their physician immediately, reported to the ethics committee with the programme discontinued. As all sessions are onsite, all interventions will be provided by allied health therapists with current and updated first aid and resuscitation certificates. All seizure management plans will be documented with parents required to bring their medications to sessions.

\section{Study procedure}

This feasibility trial is a single group study (figure 1) with three assessment time points (preintervention T1: baseline/preprogramme: 2 weeks prior to the commencement

\section{OUTCOME MEASURES}

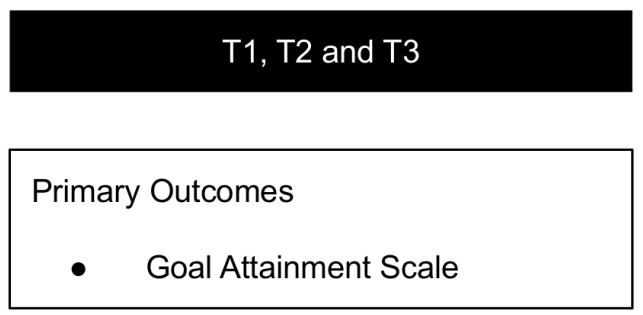

Secondary Outcomes
- $\quad$ Canadian Occupational
Performance Measure
- $\quad$ Gross Motor Function Measure
- $\quad 10$ metre walk test
- $\quad$ Peabody Developmental Motor
- Scale
- Infant and Toddler Quality of Life

Figure 1 Study design and outcome measures. WeeFIM, Functional Independence Measure. 
of the programme. T2: postrogramme: the week following the end of the 4-week programme (primary endpoint). T3: follow-up: 4 weeks from time point B (secondary endpoint). Participants will be screened for eligibility after registration of interest through an online form. The baseline T1 assessment will be completed at The Healthy Strides Foundation and once eligibility is confirmed, written consent is then obtained, and the child is scheduled to commence the programme.

\section{Demographic and classification measures}

At T1 baseline, each participant will be assessed with demographic details collected to confirm diagnosis, seizure management plan, hip status, history of botulinum neurotoxin type A injections, history of orthopaedic intervention, recent or upcoming planned hospitalisations, allergies, medication, height and weight. Each child will also be classified according to functional classification measures to include the GMFCS Expanded and Revised (for children with CP) ${ }^{66}$ the Manual Ability Classification System, ${ }^{67}$ Communication Function Classification System ${ }^{68}$ and Functional Mobility Scale. ${ }^{69}$

\section{Primary outcome measures}

Individually specific goals-GAS)

The GAS enables individualised goal setting and evaluation in areas beyond motor capacity measures and can be used for determining meaningful changes in socialisation, communication and participation. ${ }^{70} 71$ The GAS is a valid and reliable measure that is not diagnostic specific and is sensitive to detect real change within groups in paediatric research. ${ }^{70}$ The assessment consists of a five-point ordinal scale measuring outcomes from -2 (set as the baseline or starting point of how the child is currently performing) to +2 (much more than the expected outcome), with 0 being the expected outcome following intervention which indicates that the goal has been achieved. ${ }^{58}$ For this study, goals for the participants will be first established through the COPM which will be completed collaboratively between parents and the chief investigator at $\mathrm{T} 1$. The GAS enables more detail of the COPM to be objectively assessed. ${ }^{72}$ For example, a COPM goal of 'improve play skills and attention during class' may have a GAS of 'to be able to sit at a table and complete the play dough activity with verbal cues only'. The ordinal scale score is then converted to a t-score for statistical analysis and is normally distributed about a mean of 50 and an SD of 10, with a score of greater than 50 being considered clinically meaningful. ${ }^{58}$

\section{Secondary outcome measures}

Individually specific goals-COPM

The COPM is a client/family-centred valid, reliable and responsive measure for activity and participation in children with $\mathrm{CP}^{71}$ The COPM has three main areas and subareas where occupational performance problems can be identified. This includes the area of self-care (subareas include personal care, functional mobility and community management), productivity (subareas of school and play) and leisure (quiet recreation, active recreation and socialisation). A performance and satisfaction score out of 10 is obtained for each problem ( 1 being the lowest and 10 being the highest score). A change score of two or more is considered clinically significant. ${ }^{71}$

\section{Indoor walking speed-10mWT}

The $10 \mathrm{mWT}$ is a task-specific objective measure of stepping or walking speed within an indoor environment. The test can be completed both with or without a gait trainer and is not diagnostic specific. ${ }^{39} 46557374$ The $10 \mathrm{mWT}$ has excellent measurement properties. ${ }^{46}$ This measure was used in a previous study also using LTT in children with GMFCS levels III, IV and V. ${ }^{21}$ For children that cannot initiate steps within a 30 s time frame, physical facilitation for one step is provided. A maximum time of $10 \mathrm{~min}(600 \mathrm{~s})$ is provided to complete the $10 \mathrm{~m}$ and for children that cannot complete the 10 metresm, a time of $600 \mathrm{~s}$ is recorded. ${ }^{21} \mathrm{~A}$ change of $0.1 \mathrm{~m} / \mathrm{s}$ is considered to be clinically meaningful. ${ }^{26}$

\section{Burden of care-WeeFIM}

The WeeFIM has excellent measurement properties that is used to measure consistent performance of activities of daily living, functional independence and burden of care in children with disabilities. ${ }^{61}$ The WeeFIM is a semistructured interview that is guided by a specific manual to determine the level of assistance required for (1) self care; (2) transfers and mobility; (3) cognition and communication. A total of 18 items are scored on a scale of 1 (indicating total assistance required for completion of the task) to 7 (complete independence) giving a total score out of a possible $126 .{ }^{37} 38$ The WeeFIM is recommended for detecting change in activities of daily living over time in children with neurodevelopmental disabilities. ${ }^{61}$

\section{Peabody Developmental Motor Scale Version 2}

The PDMS-2 is a non-diagnostic specific assessment that is frequently used to assess motor skills. It has excellent measurement properties in children aged between 2 and 5 years with CP and is standardised and normed for children aged from birth to 6 years. ${ }^{342}$ There are three composites of the PDMS-2 that evaluate motor change (in percentage scores) following therapy and include Gross Motor, Fine Motor and Total Motor composites. The Fine Motor composite (PDMS-FM), consisting of 98 items from two subsets will be used to measure the use of small muscle systems. The two subsets of the Fine Motor composite evaluate grasp (ability to hold an object and progressing to controlled use of fingers of both hands) and visual motor integration (ability to perform complex hand-eye coordination tasks such as reach and grasping an object to build blocks and copy designs) and are scored on a 3 point criterion-referenced scale. ${ }^{62}$ The PDMS- 2 will be video-recorded and then scored by an experienced occupational therapist, blinded to assessment time point. 


\section{Sleep Disturbance Scale for Children}

The Sleep Disturbance Scale for Children (SDSC) is validated for preschool children in the measurement of sleep disorders. The questionnaire is completed by primary caregivers and explores the occurrence of sleep disorders in 26 items that are scored on a Likert scale with values ranging from 1 to 5 (with 5 representing higher severity of symptoms). A total sleep score is derived (out of 130) and correspondingly a T-score; where a T-score of more than 70 describing abnormal sleep behaviours. ${ }^{63}$ The SDSC can be used to measure previous 4 weeks of children's sleep and is a useful screening tool for evaluating comorbid sleep disorders in preschool aged children. ${ }^{63} 75$

\section{Infant and Toddler Quality of Life}

This measure was developed for infants and toddlers from 2 months of age to 5 years, adopting the WHO's definition of health. ${ }^{64}$ The survey is comprised 97 items and scored on a Likert scale based on concepts of overall health, growth and development, moods and temperaments, general behaviour and getting along and perceptions of changes in health. Items are summed and transformed on a continuum that ranges from 0 (lowest and worst possible score) to 100 (best possible score) following a standard scoring procedure. If more than half of the items of a scale are not scored by the primary caregivers, their responses will not be included in the analyses. ${ }^{64}$

\section{Gross Motor Function Measure}

Given that $\mathrm{CP}$ is the most common cause of physical disability in childhood, the GMFM will be used in children with CP only. The GMFM-66 will be used because of its high construct validity and test-retest reliability in detecting change in gross motor capacity in children with $\mathrm{CP}^{76}$ The GMFM-66 is a specific and sensitive outcome measure ${ }^{77}$ and is more sensitive when detecting change in children under 5 years of age ${ }^{76}$ Each of the 66 items will be scored based on criterion-referenced observations on a 4-point scale ${ }^{76}$ Clinically meaningful change for the GMFM-66 in children with CP aged 1.5-7 years old is 1.23 for individuals classified as GMFCS level III, and 2.88 for
GMFCS levels IV and V. ${ }^{78}$ The GMFM-66 assessment will be video recorded and scored by an experienced physiotherapist blinded to assessment time point.

\section{Semi-structured interview}

At the end of the programme, parents will be interviewed using a semi-structured interview guide based on the F-words. The purpose of the interview is to explore and understand the parent, child and family experience of the programme. The interviews will be conducted by a researcher that is not involved in the Kindy Moves intervention but has extensive experience in interviewing families of children with CP. All interviews will be conducted at Healthy Strides, in a separate room to enable privacy and audio recording (with consent). The interview guide is shown in table 1 .

\section{Kindy Moves intervention}

The dosage of the Kindy Moves intervention is 24 hours, made up of three, 2-hour sessions a week for 4 weeks. Sessions will be scheduled to ensure there are only 2 days that are consecutive, that is, Tuesday, Thursday and Friday. A maximum of four children with similar goals and age will be allocated to each group. The group setting and environmental set up of the intervention space aims to mimic a kindergarten context. Participants are able to continue with standard care during Kindy Moves.

\section{Allied health team}

The Kindy Moves allied health team will consist of physiotherapists, occupational therapists, speech pathologist, therapy assistants and undergraduate allied health student volunteers. Each child will be allocated one therapist (regardless of discipline) for each session to ensure consistency and continuity. The speech pathologist will only be involved remotely by observing videos of children's interactions during the baseline $\mathrm{T} 1$ assessment and provide communication strategies to the treating team. A review of the child's communication strategies will be videoed during a session in the second week of the programme to enable the speech pathologist to

Table 1 Key topics and prompts in the semi-structured interview guide

Prompts

\begin{tabular}{|c|c|c|}
\hline \multirow[b]{2}{*}{ Topic } & \multicolumn{2}{|l|}{ Prompts } \\
\hline & Parents & Questions \\
\hline Experience & Explain the child and parent experience in the intervention & eg, Tell me about participating in Kindy Moves \\
\hline Function & Mobility, transfers, self-care, etc & $\begin{array}{l}\text { eg, Have you noticed any changes to how your child } \\
\text { moves? }\end{array}$ \\
\hline $\begin{array}{l}\text { Contextual } \\
\text { factors }\end{array}$ & $\begin{array}{l}\text { Community-based; role of staff; interaction with other } \\
\text { families; role demands; intervention equipment }\end{array}$ & $\begin{array}{l}\text { eg, How did your involvement in Kindy Moves affect } \\
\text { your daily life? }\end{array}$ \\
\hline Impact & $\begin{array}{l}\text { Goals for child; impact on parent and family; maintaining } \\
\text { outcomes }\end{array}$ & $\begin{array}{l}\text { eg, How would you explain this programme to other } \\
\text { families? }\end{array}$ \\
\hline
\end{tabular}


adjust the recommendations for the team. Each child will subsequently have an individualised approach addressing their goals and this will be consistently reinforced by the team providing the intervention. Prior to each session, the goals of each child attending the programme will be reviewed and reinforced to ensure the team providing the intervention are focused on the individually task-specific strategies.

The 2-hour programme will be divided into three main sections to mirror activities that would occur during kindergarten. This includes morning floor time, gross motor movement and play as well as table-top activities. Each child will have their own visual schedule board so that the upcoming activities can be described to each child prior to commencing the session.

\section{Morning floor time (30 min)}

To commence the programme, a morning routine will be adopted to mirror routines at school. The floor time session will be led by a therapist or therapy assistant to set the pace of the morning routine and encourage active involvement and each child will be allocated their own therapist or therapy assistant. The routine will commence with children introducing themselves to their peers through a good morning song (with the assistance of pre-recorded audio clip of the child's name on a hand activated switch if required) followed by turn taking and choice making (through picture card options) for a song selection. Each song choice will incorporate key word signing and motor actions such as hands on head, sit to stand, clapping and dancing for commonly sung children songs including 'Five Cheeky Monkeys', 'Five Little Ducks', 'Dingle Dangle Scarecrow', 'Row-Row-Row Your Boat'. Following a song choice from each child, the floor session will conclude with a book reading. The lead therapist will encourage involvement from each child in the book reading time by pausing on pages to ask questions about what is happening or what is about to happen. Strategies to promote active involvement include hand activated switches with pre-recorded lines of the book, eye-gaze boards to enable children who are non-verbal or not able to independently turn pages to answer 'who', 'what', 'where' and 'when' questions. The same book will be used at each session to promote repetition, routine and turn taking. Individually specific gross motor goals will be incorporated into this session such as independent sitting, crawling, kneeling or standing.

\section{Gross motor movement and play through $L T$ and over-ground walking (60 min which includes donning and doffing)}

LT will be provided through partial body weight supported treadmill training with a dosage of three sets of $8 \mathrm{~min}$ with $2 \mathrm{~min}$ of standing in the harness while engaging in an upper limb activity for example, posting, throwing a ball to a target. After the $30 \mathrm{~min}$ of LT over the treadmill, over-ground walking in a gait trainer will follow for a further $20 \mathrm{~min}$. The purpose of the over-ground walking is to promote exploration and play around a busy classroom environment or during morning recess time where children can be in their gait trainers with other children. The LT and overground walking will be carried out by two therapists/ therapy assistants. The partial body weight supported treadmill training protocol is based on Behrman and Harkema (2000) ${ }^{79}$ protocol and Day et al $(2004){ }^{47}$ with standardised hand positioning during the swing and stance phase. Optimal speed is determined by establishing a spatially and temporally coordinated walking pattern $(0.8-1.5 \mathrm{~km} /$ hour $)$ with straps attached to the anterior and posterior part of the harness to optimise hip, knee and ankle kinematics during gait. Synchronisation of the timing for foot clearance and simultaneous heel strike of one limb and toe-off on the other limb for swing is provided with songs used to support timing and motivation. Ankle foot orthoses will be used if they are already prescribed for the participant as part of standard care. The duration of the session will be determined by (1) participant fatigue, (2) maintenance of step patterns and weight shift.

The over-ground walking will follow immediately after the partial body weight supported treadmill training session with children being placed in a gait trainer. Children will be encouraged to actively step, explore and play, for example, going around obstacles, play ball games or read and interact with a book. The progression of movement within the gait trainer will be dependent on individual goals and as much as possible, a hands-off approach will be adopted to promote active involvement of the child, enabling exploration and problem solving. For example, for some children the goal may be to selfpropel in a gait trainer or direct and steer themselves in a gait trainer. For children with less mobility restrictions, their progression may be for unassisted indoor walking and to negotiate obstacles.

\section{Table-top activities (30 min)}

During this session, goal directed upper limb skills will be targeted with aim to promote purposeful and task specific movements. This session will be dependent on individual goals and may include increasing the consistency of activating hand switches for play, swiping or direct access on a tablet, bilateral or bimanual hand use to complete craft, playdough, building and drawing activities. Children will be seated at a table and supported as required or as directed by the goals, for example, chair with postural support, kindergarten style school chair with feet supported or sitting on a bench without back support.

\section{Training and intervention fidelity \\ Training fidelity}

All physiotherapists and occupational therapists will be registered under the Australian Health Practitioner Regulation Agency and the speech pathologist registered under Speech Pathology Australia. All therapists and therapy assistants have credentialed 
competency in the provision of the intervention (LT facilitation, set up of as well as donning and doffing into the harness and gait trainer). This is an annual competency that is signed off by the chief investigator. The chief investigator will complete all COPM having completed the online COPM training module. The GMFM will be videoed and assessed by a physiotherapist with extensive experience in GMFM assessments having completed the training prior (noting it is no longer available). All therapists and undergraduate allied health volunteers will complete an 8-hour training programme on the Kindy Moves intervention. The training will include key word signing, knowledge of all songs and corresponding key word sign, use of communication boards, programming hand activated switches for toys and audio recordings and LT support and facilitation. Only allied health students who have passed the competency standards can support the provision of the intervention.

\section{Intervention fidelity}

Several strategies will be undertaken to ensure fidelity of the intervention.

- Training sessions for all therapists and therapy assistants with set competency standards that need to be demonstrated and passed by the chief investigator.

- All children attending the programme will have their own individualised programme outlining the goals and strategies.

- Planning session prior to the commencement of a programme for all individual strategies to be discussed among the treating team and chief investigator. The framework for the planning sessions will be in line with the functional therapy guidelines. ${ }^{22}$

- Stand-up meeting prior to each session to review the goals of each child, feedback from prior session and reinforce child specific strategies.

- Where possible, the same therapist or therapy assistant will be with the child in the session to ensure consistency within the session.

\section{Consumer involvement}

The design of the intervention (including the dosage, scheduling of sessions, individualised sessions within a group setting) and selection of outcome measures was not only directed by current published evidence but also from the input of parents and therapists from a previous qualitative feasibility study of intensive LT in children with CP functioning that were either marginally ambulant or non-ambulant, aged between 5 and 12 years (awaiting publication). In addition to this, the Healthy Strides Advisory Research Group which includes consumer representatives (parents of children with CP under 10 years of age) were part of the planning and development of the study protocol and intervention.

\section{Participant and data management}

The number of self-referrals, screened to be eligible, offered placements and those not proceeding with the programme will be recorded. Progress notes regarding session progress, intervention dosage or reported adverse events and attendance will be completed after each session throughout the study period. In case of study withdrawal or loss to follow-up, intention to treat will be applied. All data will be electronic including signed consent forms, assessment forms and video recordings of assessments accessible only to the study team with two stage password access at The Healthy Strides Foundation's secure database. Identification codes will be allocated to the GMFM and PDMS-2 assessment due to the blinded assessor. These codes will be generated by another investigator using a random number allocation sequence so that the time point of the video recording cannot be identified.

\section{Statistical methods}

The assumption of normality will be tested for all measures through examining distributional plots, Q-plots and the Shapiro-Wilk test. For data normally distributed, parametric tests will be applied with means and SD for each group at each assessment time point reported. For ordinal data, or where data are not normally distributed despite transformations, nonparametric tests will be applied with medians and IQRs reported. Intention to treat analysis will be applied. Authors MH and DP will individually categorise the GAS and COPM according to the Family of Participation Related Constructs (fPRC)..$^{80}$

An Analysis of Covariance (ANCOVA) will be used to determine group mean differences and 95\% CIs, with statistical significance being set at $\mathrm{p}<0.05$. Following GAS classification, mean differences in T-scores will also be determined for the activity and participation-based goals as classified by the fPRC. Clinically significant changes (for the GAS and COPM) will be reported as a percentage of goals achieved and not achieved. Attendance rates will be tallied based on attendance sheets from progress notes and the group mean attendance established as a proportion of 12 possible sessions attended. No interim analysis will occur with data only analysed at the conclusion of the trial (with 40 participants recruited).

\section{Qualitative analysis}

The interviews will be transcribed verbatim with all identifiable features such as names removed and replaced with pseudonyms. After reading the transcripts multiple times, data will be analysed thematically using an open coding process to identify meaning units. After applying the open coding framework, meaning units will be categorised into themes and grouped into higher order categories. This process will be completed by two reviewers, enabling comparisons and connections between themes to be explored within the context 
of the F-words. ${ }^{57}$ Several methods of trustworthiness will be undertaken, including credibility (through member checking), credibility through a critical friends approach, transferability through purposive sampling and dependability through overlap methods with triangulation of data with the quantitative measures. ${ }^{81-83}$

\section{DISCUSSION}

This paper outlines the protocol and background for establishing the feasibility of an intensive activity-based intervention on goal attainment and motor capacity delivered within an interdisciplinary framework for children with CP and CP like conditions functioning with GMFCS levels III, IV and V (or equivalent to if non-CP). The intervention is designed to meet the individual needs of school readiness for children with $\mathrm{CP}$ and CP like conditions. Outcome measures have been selected to represent the ICF-CY domains. We hope that the findings from this research will be published and disseminated in a peer-reviewed journal. Individualised adaptations will be necessary to ensure the child's individual goals are met, However, every effort will be made to standardise each element of the intervention. The intervention is comprised several elements in order to meet the multiple key skill areas of school readiness. This is a limitation of the intervention as it will not be possible to differentiate between the effects of each of the individual elements.

\section{Ethics and dissemination}

Kindy Moves has been approved by the Human Research Ethics Committee of Curtin University. Participant information will be provided to all participants prior to entry into the study. Written and informed consent will be obtained from all participants.

Knowledge translation will be guided by the Knowledge Translation Planning Template. ${ }^{84}$ Project partners include researchers, consumers and practitioners who will be supported by the project investigators. Specific knowledge translation strategies will be targeted throughout the Kindy Moves project, in partnership with our stakeholders. This will include any peer-reviewed publications, plain language summaries (digital and written), media case studies and conference presentations.

\section{Author affiliations}

${ }^{1}$ School of Allied Health, Curtin University, Perth, Western Australia, Australia

${ }^{2}$ The Healthy Strides Foundation, Perth, Western Australia, Australia

${ }^{3}$ Telethon Institute for Child Health Research, Nedlands, Western Australia, Australia

\section{Twitter Dayna Pool @daynapool}

Acknowledgements The authors acknowledge the Healthy Strides Research Advisory Council for consulting and providing guidance on the development of the intervention.

Collaborators The Healthy Strides Research Advisory Council comprises of the Healthy Strides clinical team: Loren West, Corrin Walmsley, Georgia Hoffman, Marissa Smith, Eddie Pool, Meagan Smith, Georgina Jones, Matthew Haddon, Jordan Dinh and Bridget Chapman. Research Advisors: Dr Ashleigh Thornton, Dr
Sue McCabe and Dr Claire Willis. Consumer Advisors: Ben O'Rourke, Noraishah Naim, Alishum Osman Ali.

Contributors All authors meet the ICMJE criteria for authorship, making substantial contributions to the study design, drafting the manuscript and proofing the final version for submission. DP conceptualised, planned, developed and wrote the study protocol. CE conceptualised and wrote the study protocol.

Funding This research was supported by the Telethon7 Trust 2019 and 2020. Competing interests None declared.

Patient and public involvement Patients and/or the public were involved in the design, or conduct, or reporting, or dissemination plans of this research. Refer to the Methods section for further details.

Patient consent for publication Not required.

Provenance and peer review Not commissioned; externally peer reviewed.

Open access This is an open access article distributed in accordance with the Creative Commons Attribution Non Commercial (CC BY-NC 4.0) license, which permits others to distribute, remix, adapt, build upon this work non-commercially, and license their derivative works on different terms, provided the original work is properly cited, appropriate credit is given, any changes made indicated, and the use is non-commercial. See: http://creativecommons.org/licenses/by-nc/4.0/.

ORCID iD

Dayna Pool http://orcid.org/0000-0001-8313-5661

\section{REFERENCES}

1 Human Early Learning Partnership \& Commission on Social Determinants of Health. Early child development : a powerful equalizer: final report for the World Health Organization's Commission on the Social Determinants of Health. / Prepared by Arjumand Siddiqi, Lori G. Irwin, Dr. Clyde Hertzman. Vancouver: Human Early Learning Partnership, 2007.

2 Heckman JJ, Masterov DV. The productivity argument for investing in young children. Rev Agri Econom 2007;29:446-93.

3 Nores M, Barnett WS. Benefits of early childhood interventions across the world: (under) investing in the very young. Econ Educ Rev 2010;29:271-82.

4 Richter LM, Daelmans B, Lombardi J, et al. Investing in the foundation of sustainable development: pathways to scale up for early childhood development. Lancet 2017;389:103-18.

5 Goldfeld S, O'Connor E, O'Connor M, et al. The role of preschool in promoting children's healthy development: Evidence from an Australian population cohort. Early Child Res Q 2016;35:40-8.

6 Roberts G, Lim J, Doyle LW, et al. High rates of school readiness difficulties at 5 years of age in very preterm infants compared with term controls. J Dev Behav Pediatr 2011;32:117-24.

7 Gehrmann FE, Coleman A, Weir KA, et al. School readiness of children with cerebral palsy. Dev Med Child Neurol 2014;56:786-93.

8 Cairney J, Hay JA, Faught BE, et al. Developmental coordination disorder, generalized self-efficacy toward physical activity, and participation in organized and free play activities. J Pediatr 2005;147:515-20.

9 Van Hus JW, Potharst ES, Jeukens-Visser M, et al. Motor impairment in very preterm-born children: links with other developmental deficits at 5 years of age. Dev Med Child Neurol 2014;56:587-94.

10 Report of the Australian cerebral palsy register, birth years 19932009, 2016.

11 Palisano R, Rosenbaum P, Walter S, et al. Development and reliability of a system to classify gross motor function in children with cerebral palsy. Dev Med Child Neurol 1997;39:214-23.

12 Palisano RJ, Hanna SE, Rosenbaum PL, et al. Validation of a model of gross motor function for children with cerebral palsy. Phys Ther 2000;80:974-85.

13 World Health Organization. Neurological disorders: public health challenges, 2006. Available: https://www.who.int/mental_health/ neurology/neurological_disorders_report_web.pdf [Accessed 9 Nov 2020].

14 Smithers-Sheedy H, Badawi N, Blair E, et al. What constitutes cerebral palsy in the twenty-first century? Dev Med Child Neurol 2014;56:323-8.

15 Novak I, Honan I. Effectiveness of paediatric occupational therapy for children with disabilities: a systematic review. Aust Occup Ther J 2019;66:258-73.

16 Ostensjø S, Carlberg EB, Vøllestad NK. Everyday functioning in young children with cerebral palsy: functional skills, caregiver 
assistance, and modifications of the environment. Dev Med Child Neurol 2003;45:603-12

17 Cope S, Mohn-Johnsen S. The effects of dosage time and frequency on motor outcomes in children with cerebral palsy: a systematic review. Dev Neurorehabil 2017;20:376-87.

18 Bleyenheuft Y, Gordon AM. Hand-arm bimanual intensive therapy including lower extremities (HABIT-ILE) for children with cerebral palsy. Phys Occup Ther Pediatr 2014;34:390-403.

19 Størvold GV, Jahnsen RB, Evensen KAl, et al. Factors associated with enhanced gross motor progress in children with cerebral palsy: a register-based study. Phys Occup Ther Pediatr 2018;38:548-61.

20 Jackman M, Lannin N, Galea C, et al. What is the threshold dose of upper limb training for children with cerebral palsy to improve function? A systematic review. Aust Occup Ther J 2020;67:269-80.

21 Pool D, Valentine J, Taylor NF, et al. Locomotor and robotic assistive gait training for children with cerebral palsy. Dev Med Child Neurol 2021;63:328-35

22 Geijen M, Ketelaar M, Sakzewski L, et al. Defining functional therapy in research involving children with cerebral palsy: a systematic review. Phys Occup Ther Pediatr 2020;40:231-46.

23 Novak I, Morgan C, Fahey M, et al. State of the evidence traffic lights 2019: systematic review of interventions for preventing and treating children with cerebral palsy. Curr Neurol Neurosci Rep 2020;20:3.

24 Jeglinsky I, Salminen A-L, Carlberg EB, et al. Rehabilitation planning for children and adolescents with cerebral palsy. J Pediatr Rehabil Med 2012;5:203-15.

25 Choi BCK, Pak AWP. Multidisciplinarity, interdisciplinarity and transdisciplinarity in health research, services, education and policy: 1. definitions, objectives, and evidence of effectiveness. Clin Invest Med 2006;29:351-64.

26 Soper AK, Cross A, Rosenbaum P, et al. Knowledge translation strategies to support service providers' implementation of the "Fwords in Childhood Disability". Disabil Rehabil 2020;45:1-7.

27 Jan MMS. Cerebral palsy: comprehensive review and update. Ann Saudi Med 2006;26:123-32.

28 Trabacca A, Russo L, Losito L, et al. The ICF-CY perspective on the neurorehabilitation of cerebral palsy: a single case study. J Child Neurol 2012;27:183-90.

29 Glader L, Plews-Ogan J, Agrawal R. Children with medical complexity: creating a framework for care based on the International classification of functioning, disability and health. Dev Med Child Neurol 2016;58:1116-23.

30 Morgan C, Novak I, Dale RC, et al. Single blind randomised controlled trial of GAME (Goals - Activity - Motor Enrichment) in infants at high risk of cerebral palsy. Res Dev Disabil 2016;55:256-67.

31 Danger S, Landreth G. Child-centered group play therapy with children with speech difficulties. Int J Play Ther 2005;14:81-102.

32 Astramovich RL, Lyons C, Hamilton NJ. Play therapy for children with intellectual disabilities. J Child Adolesc Couns 2015;1:27-36.

33 Fauconnier J, Dickinson HO, Beckung E, et al. Participation in life situations of 8-12 year old children with cerebral palsy: cross sectional European study. BMJ 2009;338:b1458

34 Michelsen SI, Flachs EM, Uldall P, et al. Frequency of participation of 8-12-year-old children with cerebral palsy: a multi-centre crosssectional European study. Eur J Paediatr Neurol 2009;13:165-77.

35 Imms C. Children with cerebral palsy participate: a review of the literature. Disabil Rehabil 2008;30:1867-84.

36 Bleyenheuft Y, Arnould C, Brandao MB, et al. Hand and arm bimanual intensive therapy including lower extremity (HABIT-ILE) in children with unilateral spastic cerebral palsy: a randomized trial. Neurorehabil Neural Repair 2015;29:645-57.

37 Mutlu A, Krosschell K, Spira DG. Treadmill training with partial bodyweight support in children with cerebral palsy: a systematic review. Dev Med Child Neurol 2009;51:268-75.

38 Peterson MD, Gordon PM, Hurvitz EA. Chronic disease risk among adults with cerebral palsy: the role of premature sarcopoenia, obesity and sedentary behaviour. Obes Rev 2013;14:171-82.

39 Willoughby KL, Dodd KJ, Shields N. A systematic review of the effectiveness of treadmill training for children with cerebral palsy. Disabil Rehabil 2009;31:1971-9.

40 Anderson DI, Campos JJ, Witherington DC, et al. The role of locomotion in psychological development. Front Psychol 2013;4:440.

41 Huang $\mathrm{H}-\mathrm{H}$, Chen $\mathrm{C}-\mathrm{L}$. The use of modified ride-on cars to maximize mobility and improve socialization-a group design. Res Dev Disabil 2017;61:172-80

42 Ryan JM, Cassidy EE, Noorduyn SG, et al. Exercise interventions for cerebral palsy. Cochrane Database Syst Rev 2017;2017.

43 Fonzo M, Sirico F, Corrado B. Evidence-Based physical therapy for individuals with Rett syndrome: a systematic review. Brain Sci 2020;10:410.
44 Wheeler AC, Sacco P, Cabo R. Unmet clinical needs and burden in Angelman syndrome: a review of the literature. Orphanet J Rare Dis 2017;12:164

45 Willoughby KL, Dodd KJ, Shields N, et al. Efficacy of partial body weight-supported treadmill training compared with overground walking practice for children with cerebral palsy: a randomized controlled trial. Arch Phys Med Rehabil 2010;91:333-9.

46 Dodd KJ, Foley S. Partial body-weight-supported treadmill training can improve walking in children with cerebral palsy: a clinical controlled trial. Dev Med Child Neurol 2007;49:101-5.

47 Day JA, Fox EJ, Lowe J, et al. Locomotor training with partial body weight support on a treadmill in a nonambulatory child with spastic tetraplegic cerebral palsy: a case report. Pediatr Phys Ther 2004;16:106-13.

48 Schindl MR, Forstner $\mathrm{C}$, Kern $\mathrm{H}$, et al. Treadmill training with partial body weight support in nonambulatory patients with cerebral palsy. Arch Phys Med Rehabil 2000;81:301-6.

49 Verschuren O, Helders PJM, Mattern-Baxter K. Effects of intensive locomotor treadmill training on young children with cerebral palsy. Pediatr Phys Ther 2009;21:319-19.

50 Valentín-Gudiol M, Mattern-Baxter K, Girabent-Farrés M, et al. Treadmill interventions in children under six years of age at risk of neuromotor delay. Cochrane Database Syst Rev 2017;7:Cd009242.

51 Ginsburg KR, American Academy of Pediatrics Committee on Communications, American Academy of Pediatrics Committee on Psychosocial Aspects of Child and Family Health. The importance of play in promoting healthy child development and maintaining strong parent-child bonds. Pediatrics 2007;119:182-91.

52 Novak I, Mclntyre S, Morgan C, et al. A systematic review of interventions for children with cerebral palsy: state of the evidence. Dev Med Child Neurol 2013;55:885-910.

53 Patel DR. Therapeutic interventions in cerebral palsy. Indian J Pediatr 2005;72:979-83.

54 Mickan SM. Evaluating the effectiveness of health care teams. Aust Health Rev 2005;29:211-7.

55 Damiano DL, DeJong SL. A systematic review of the effectiveness of treadmill training and body weight support in pediatric rehabilitation. J Neurol Phys Ther 2009;33:27-44

56 Bowen DJ, Kreuter M, Spring B, et al. How we design feasibility studies. Am J Prev Med 2009;36:452-7.

57 Rosenbaum P, Gorter JW. The 'F-words' in childhood disability: I swear this is how we should think! Child Care Health Dev 2012;38:457-63.

58 Turner-Stokes L. Goal attainment scaling (GAS) in rehabilitation: a practical guide. Clin Rehabil 2009;23:362-70.

59 Carswell A, McColl MA, Baptiste S, et al. The Canadian occupational performance measure: a research and clinical literature review. Can J Occup Ther 2004;71:210-22.

60 Booth ATC, Buizer Al, Meyns P, et al. The efficacy of functional gait training in children and young adults with cerebral palsy: a systematic review and meta-analysis. Dev Med Child Neurol 2018;60:866-83.

61 Ottenbacher KJ, Msall ME, Lyon N, et al. The WeeFIM instrument: its utility in detecting change in children with developmental disabilities. Arch Phys Med Rehabil 2000;81:1317-26.

62 Wang $\mathrm{H}-\mathrm{H}$, Liao H-F, Hsieh C-L. Reliability, sensitivity to change, and responsiveness of the peabody developmental motor scales-second edition for children with cerebral palsy. Phys Ther 2006;86:1351-9.

63 Romeo DM, Brogna C, Musto E, et al. Sleep disturbances in preschool age children with cerebral palsy: a questionnaire study. Sleep Med 2014;15:1089-93.

64 Spuijbroek AT, Oostenbrink R, Landgraf JM, et al. Health-related quality of life in preschool children in five health conditions. Qual Life Res 2011;20:779-86.

65 Bleyenheuft Y, Ebner-Karestinos D, Surana B, et al. Intensive upperand lower-extremity training for children with bilateral cerebral palsy: a quasi-randomized trial. Dev Med Child Neurol 2017:59:625-33.

66 Palisano RJ, Rosenbaum P, Bartlett D, et al. Content validity of the expanded and revised gross motor function classification system. Dev Med Child Neurol 2008;50:744-50.

67 Eliasson A-C, Krumlinde-Sundholm L, Rösblad B, et al. The manual ability classification system (MACS) for children with cerebral palsy: scale development and evidence of validity and reliability. Dev Med Child Neurol 2006;48:549-54.

68 Hidecker MJC, Cunningham BJ, Thomas-Stonell N, et al. Validity of the communication function classification system for use with preschool children with communication disorders. Dev Med Child Neurol 2017;59:526-30.

69 Graham HK, Harvey A, Rodda J, et al. The functional mobility scale (FMS). J Pediatr Orthop 2004;24:514-20. 
70 Livingstone R, Paleg G. Measuring outcomes for children with cerebral palsy who use gait trainers. Technology 2016;4:1-19.

71 Cusick A, Mclntyre S, Novak I, et al. A comparison of goal attainment scaling and the Canadian occupational performance measure for paediatric rehabilitation research. Pediatr Rehabil 2006;9:149-57.

72 Novak I, Cusick A, Lannin N. Occupational therapy home programs for cerebral palsy: double-blind, randomized, controlled trial. Pediatrics 2009;124:e606-14.

73 Meyer-Heim A, Borggraefe I, Ammann-Reiffer C, et al. Feasibility of robotic-assisted locomotor training in children with central gait impairment. Dev Med Child Neurol 2007;49:900-6.

74 Mattern-Baxter K. Effects of partial body weight supported treadmill training on children with cerebral palsy. Pediatr Phys Ther 2009;21:12-22.

75 Romeo DM, Bruni O, Brogna C, et al. Application of the sleep disturbance scale for children (SDSC) in preschool age. Eur $J$ Paediatr Neurol 2013;17:374-82.

76 Russell DJ, Avery LM, Rosenbaum PL, et al. Improved scaling of the gross motor function measure for children with cerebral palsy: evidence of reliability and validity. Phys Ther 2000;80:873-85.

77 Wang $\mathrm{H}-\mathrm{Y}$, Yang $\mathrm{YH}$. Evaluating the responsiveness of 2 versions of the gross motor function measure for children with cerebral palsy. Arch Phys Med Rehabil 2006;87:51-6.
78 Ko J. Sensitivity to functional improvements of GMFM-88, GMFM66 , and PEDI mobility scores in young children with cerebral palsy. Percept Mot Skills 2014;119:305-19.

79 Behrman AL, Harkema SJ. Spinal Cord Injury Special Series Locomotor Training After Human Spinal Cord Injury : A Series of Case Studies. Physical Therapy 2000;80:688-700.

80 Imms C, Granlund M, Wilson PH, et al. Participation, both a means and an end: a conceptual analysis of processes and outcomes in childhood disability. Dev Med Child Neurol 2017;59:16-25.

81 Guba EG. Criteria for assessing the trustworthiness of naturalistic inquiries. Educ Comm Technol J 1981;29:75-91.

82 Smith B, McGannon KR. Developing rigor in qualitative research: problems and opportunities within sport and exercise psychology. Int Rev Sport Exerc Psychol 2018;11:101-21.

83 Portney LG, Watkins MP. Foundations of clinical research: applications to practice. 3rd edn. New Jersey: Person Prentice Hall, 2009.

84 Barwick M. Building scientist capacity in knowledge translation: development of the knowledge translation planning template. Technol Innov Manage Rev 2016;6:9-15. 